\title{
Ab Initio Quantum Chemistry using the Density Matrix Renormalization Group
}

\author{
Steven R. White ${ }^{1}$ and Richard L. Martin ${ }^{2}$ \\ ${ }^{1}$ Department of Physics and Astronomy, University of California, Irvine, CA 92697 \\ 2 Theoretical Division, MSB268, Los Alamos National Laboratory, Los Alamos, NM 87545
}

(September 24, 2018)

\begin{abstract}
In this paper we describe how the density matrix renormalization group (DMRG) can be used for quantum chemical calculations for molecules, as an alternative to traditional methods, such as configuration interaction or coupled cluster approaches. As a demonstration of the potential of this approach, we present results for the $\mathrm{H}_{2} \mathrm{O}$ molecule in a standard gaussian basis. Results for the total energy of the system compare favorably with the best traditional quantum chemical methods.
\end{abstract}

PACS Numbers: 31.15.Ar, 71.15.-m, 31.25.Eb

Since its development in 1992 [1], the density matrix renormalization group (DMRG) has become one of the most widely used numerical techniques for simulations of one dimensional quantum lattice systems. For systems with short ranged interactions, the calculation time for DMRG grows only linearly with the length of the system, while the errors usually decrease exponentially with the calculation time [2]. Consequently, very high accuracy results are possible even on very large systems.

Most applications of DMRG have been to lattice models for strongly correlated systems. Recently, Fano, Ortolani, and Ziosi applied DMRG to a Pariser-Parr-Pople (PPP) Hamiltonian for a cyclic polyene [3]. The PPP model is more realistic than many models in that long range coulomb interactions are included. Fano, et. al. found that DMRG compared quite favorably to coupled cluster approximations. Here, we go a step further: we consider the application of DMRG to the fully ab initio determination of the electronic structure of atoms and molecules. The successful adaptation of DMRG to this field could potentially open up a wide range of improved calculational techniques, characterized by high accuracy and improved scaling of calculation time with system size. As a first step in this direction, we show here that DMRG can be successfully used to obtain very accurate many-body solutions for small molecules.

We will use DMRG within the conventional quantum chemical framework of a finite basis set with nonorthogonal basis functions made from products of gaussian radial functions and Cartesian harmonics centered on each atom. The initial step of the calculation is a standard Hartree Fock (HF) calculation in which a Hamiltonian is produced within the orthogonal HF basis. DMRG is then used as a procedure for including correlations beyond $\mathrm{HF}$, much as the configuration interaction (CI) or coupled cluster methods are used.

Within the HF basis, the Hamiltonian is in principle no different from other model Hamiltonians which have been studied using DMRG. It can be written as

$$
H=\sum_{i j \sigma} T_{i j} c_{i \sigma}^{\dagger} c_{j \sigma}+\frac{1}{2} \sum_{i j k l \sigma \sigma^{\prime}} V_{i j k l} c_{i \sigma}^{\dagger} c_{j \sigma^{\prime}}^{\dagger} c_{k \sigma^{\prime}} c_{l \sigma} .
$$

Here $T_{i j}$ contains the electron kinetic energy and the Coulomb interaction between the electrons and the nuclei, while $V_{i j k l}$ describes the electron-electron Coulomb interaction. The most important difference in this Hamiltonian from model Hamiltonians is the large number of interaction terms $V_{i j k l}: N^{4}$, where $N$ is the number of basis functions or orbitals. The number of electrons, $N_{\mathrm{el}}$, is less important in a DMRG calculation. The large number of terms makes standard DMRG programs very inefficient, and below we describe procedures for improving the efficiency of the treatment of these terms 河.

In our approach, an ordering of the orbitals is chosen, and each orbital is treated as a "site" in a onedimensional lattice. Since this arrangement is artificial, the Hamiltonian is long-ranged. The orbitals can be sorted according to various criteria. We have found that sorting them in order to minimize strong interactions between widely separated orbitals is probably best, but they can also be arranged by HF orbital energy.

Once this ordering is chosen, a standard DMRG finitesystem algorithm can be used [1]. In this procedure, collections of orbitals are represented as "blocks". The properties of a block are defined by listing the many-body states of the block and by storing matrices representing operators acting on that collection of orbitals. The representation is approximate, since not all of the many-body states are retained. For example, if the block happened to represent the $N_{\mathrm{el}} / 2$ occupied HF orbitals, then a reasonable set of states to represent that block would consist of one "filled" state with $N_{\text {el }}$ electrons, $N_{\text {el }}$ one-hole states with $N_{\mathrm{el}}-1$ electrons, and $\left(N_{\mathrm{el}}^{2}-N_{\mathrm{el}}\right) / 2$ two-hole states with $N_{\mathrm{el}}-2$ electrons. Assuming the one- and two-particle states were represented in the "unoccupied" block, this set of states would allow all singly and doubly excited configurations to be formed, but would leave out all higher excitations. DMRG is an iterative procedure, in which at each step there are two blocks, with all the orbitals belonging to one of the two blocks [5]. Iterations, or sweeps, involve transfering orbitals one at a time from the right to the left block, until the right block has only one orbital, and then reversing the direction. At 
each step, a new set of states is chosen to represent the block. The number of states $m$ kept per block controls the accuracy of the calculation, as well as the storage and computation time.

However, rather than one having to choose the manybody states which describe a block, the states are chosen in an optimal way by DMRG as the eigenstates of a many-particle density matrix. This procedure is somewhat related to the use of a single particle density matrix to choose natural orbitals in quantum chemistry. However, here the many particle states are much more complicated - too complicated, for a system of reasonable size, to represent in terms of a single particle basis. Instead, the states are described in terms of the matrix elements of various operators between these states. The complicated form of the many particle basis allows much more rapid convergence in the number of states $m$ than in a configuration expansion.

The operators which describe a block are chosen in order to be able to generate the Hamiltonian operator for the system. For example, in order to construct the kinetic energy, we must keep matrix elements for the operators $c_{i \sigma}^{\dagger}$, for all orbitals $i$ in the block. These operators allow us to construct terms $c_{i \sigma}^{\dagger} c_{j \sigma}$ where $j$ is not in the block. In addition, we must keep matrices for $c_{i \sigma}^{\dagger} c_{j \sigma}$ if both $i$ and $j$ are in the block. Note that one cannot avoid storing a matrix for $A B$ simply because one has stored matrices for $A$ and $B$ : the incomplete nature of the basis means that the matrix for $A B$ is not the product of the matrices for $A$ and $B$. In order to describe the Coulomb interaction, it appears that $o\left(N^{4}\right)$ operators of the form $c_{i \sigma}^{\dagger} c_{j \sigma^{\prime}}^{\dagger} c_{k \sigma^{\prime}} c_{l \sigma}$, must be kept, where $i, j, k$, and $l$ are all in the block. In addition, $o\left(N^{3}\right)$ additional operators are needed to construct terms when some of the $i j k l$ are not in the block.

Although it appears that $o\left(N^{4}\right)$ operator matrices must be stored per block, a completely standard DMRG "trick" reduces this number to $o\left(N^{3}\right)$. The trick is to sum terms together into a single block Hamiltonian matrix once all of the parts of the term are in the block. Hence there is no need to store terms of the form $c_{i \sigma}^{\dagger} c_{j \sigma^{\prime}}^{\dagger} c_{k \sigma^{\prime}} c_{l \sigma}$; these terms are multiplied by $V_{i j k l}$ and summed into $H$. In a typical model Hamiltonian, this tricks reduces the number of operators stored per block from $o(N)$ to $o(1)$. Here, we still have $o\left(N^{3}\right)$ operators. Since $o(N)$ blocks must be stored, the storage is $o\left(N^{4} m^{2}\right)$.

Additional improvements can be made by combining other operators, as was first done by Xiang in adapting DMRG to momentum space calculations $[6]$. There are $o\left(N^{3}\right)$ operators with three $c$ and $c^{\dagger}$ operators, used to construct the Coulomb interaction. These can be largely eliminated by constructing complementary operators like

$$
O_{i \sigma}=\sum_{j k l \sigma^{\prime}} V_{i j k l} c_{j \sigma^{\prime}}^{\dagger} c_{k \sigma^{\prime}} c_{l \sigma}
$$

The corresponding parts of the Coulomb interaction can be constructed as

$$
\sum_{i \sigma} c_{i \sigma}^{\dagger} O_{i \sigma}
$$

This trick reduces $o\left(N^{3}\right)$ operators to $o(N)$.

At this point, the dominant terms remaining are $o\left(N^{2}\right)$ operators with two $c$ and $c^{\dagger} \mathrm{s}$. The total storage for these, $o\left(N^{3} m^{2}\right)$, is now manageable. Additional complementary operators can reduce computation time, however. The dominant part of a DMRG calculation is the iterative diagonalization of the Hamiltonian of the system, which is done once per step, or $\sim N$ times per sweep. The dominant part of this is the multiplication of a vector by Hamiltonian terms of the form

$$
\sum_{i j \in L} \sum_{k l \in R} V_{i j k l}\left[c_{i}^{\dagger} c_{j}^{\dagger}\right]\left[c_{k} c_{l}\right]
$$

plus other combinations where, for example, $i$ and $l$ belong to the left block $L, j$ and $k$ to the right block $R$. Here [] denote the matrix for the corresponding operator. There are $o\left(N^{4}\right)$ such terms; multiplication of a vector by these terms requires $o\left(N^{4} m^{3}\right)$ operations. To reduce this computation time, we construct complementary operators

$$
O_{i j}^{R}=\sum_{k l \in R} V_{i j k l}\left[c_{k} c_{l}\right] \quad \forall i j \in L
$$

plus other two operator combinations corresponding to other orbitals being in the left block. Constructing these operators at each step requires $o\left(N^{4} m^{2}\right)$ operations. However, this is not necessary: one can save these operators from the previous step, transform them to the current basis, and add in the additional terms coming from the new site being added to the block, in $o\left(N^{2} m^{3}\right)+o\left(N^{3} m^{2}\right)$ operations. Using the complementary operators, corresponding Hamiltonian terms become

$$
\sum_{i j \in L}\left[c_{i}^{\dagger} c_{j}^{\dagger}\right] O_{i j}^{R}
$$

plus similar terms. The calculation time to multiply a vector by these terms is $o\left(N^{2} m^{3}\right)$, giving $o\left(N^{3} m^{3}\right)$ per DMRG sweep.

After all of these optimizations are used, the final calculation time for the whole calculation is $o\left(N^{3} m^{3}\right)+$ $o\left(N^{4} m^{2}\right)$. The final storage is $o\left(N^{3} m^{2}\right)$, but only $o\left(N^{2} m^{2}\right)$ needs to be in RAM; the rest can be on disk with little cost in calculation time. The time for the initial Hartree Fock calculation is $o\left(N^{4}\right)$, which is neglible in comparison. In the test calculations below, the number of states kept per block $m$ is typically a few hundred, and $N=25$. However, in cases such as linear chain molecules, we expect to be able to hold $m$ constant as the the length of the system increases, so that 
eventually $N \sim m$. If high accuracy was not required, one could have $N>>m$. Note that even $m=1$ gives results slightly more accurate than Hartree Fock. If a localized basis, rather than the Hartree Fock basis, were used in a large molecule, so that many coefficients $V_{i j k l}$ were neglible, then the calculation time could potentially be reduced to $o\left(N^{2} m^{3}\right)$. If, in addition, the long range part of the Coulomb interaction could be neglected, as is done in many model Hamiltonians, or closely approximated with a multipole expansion, the calculation time would be $o\left(\mathrm{Nm}^{3}\right)$. However, in the current ab initio calculations we are far from this regime.

As a test case, we have studied a water molecule in a standard basis, comparing with the benchmark full configuration interaction calculations of Bauschlicher and Taylor [7. In this work, exact results for the $\mathrm{H}_{2} \mathrm{O}$ molecule within a particular basis were compared with various approximate approaches. (In the reference calculations and our work, the innermost $\mathrm{O}$ orbital was "frozen".) We have used the same basis for the calculations here. In Fig. 1, we show DMRG results compared with Hartree Fock and singles and doubles configuration interaction (SDCI), for the molecule in its equilibrium geometry. Both DMRG and SDCI results are variational. The DMRG results become more accurate than the SDCI for $m \approx 70$.

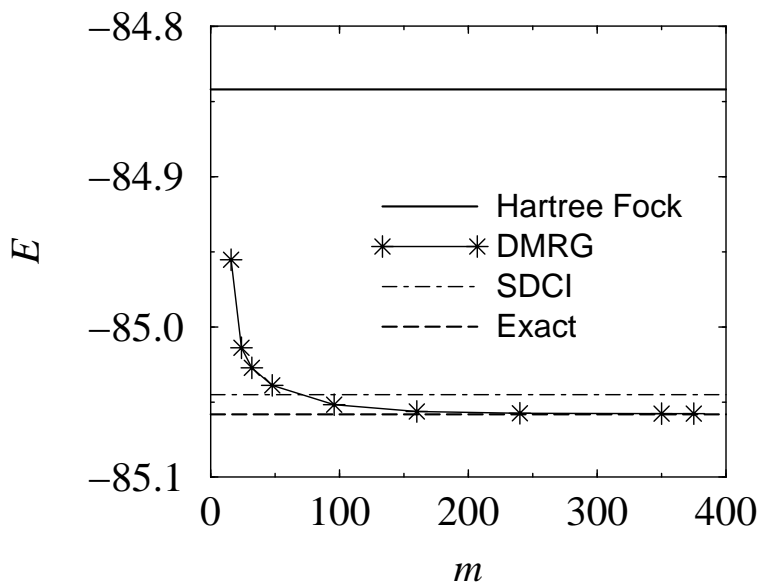

FIG. 1. Ground state energy of a water molecule in a 25 orbital basis using various methods. DMRG results are as a function of the number of states kept per block $m$; other results, which have no $m$ dependence, are plotted as horizontal lines. Energies are in Hartrees.

In Fig. 2, we show results on an expanded scale for the same system. We also compare with multireference configuration interaction calculations (MRCI), and MRCI plus an estimated correction (the Davidson correction) (MRCI+Q). MRCI is variational, but $\mathrm{MRCI}+\mathrm{Q}$ is not. We see that DMRG becomes more accurate than MRCI for $m \approx 110$, and more accurate than MRCI+Q for $m \approx 200$. The most accurate DMRG result is off by 0.00024 Hartrees. The MRCI+Q results were the most accurate of the approximate results reported by Bauschlicher and Taylor, with an error of 0.0014 .

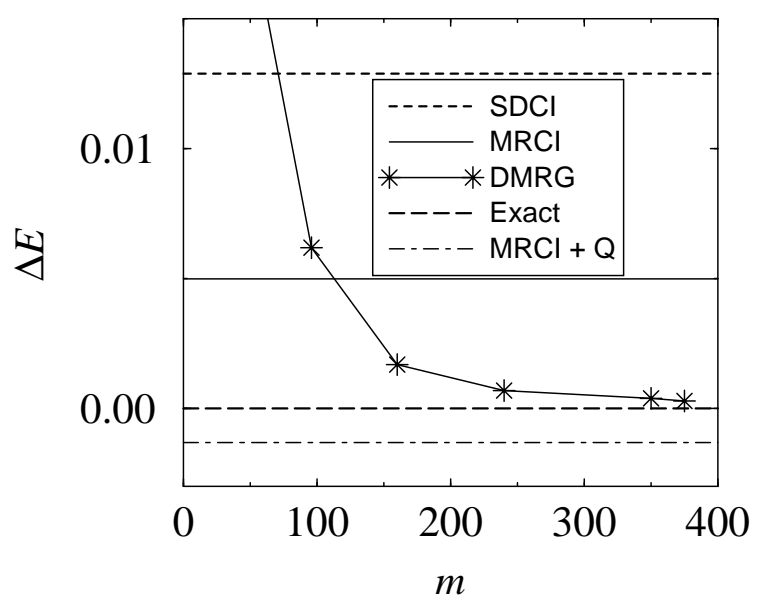

FIG. 2. Difference between the exact ground state energy within the given basis, and the approximate energy for various approaches. The system and basis is the same as in Fig. 1. Energies are in Hartrees.

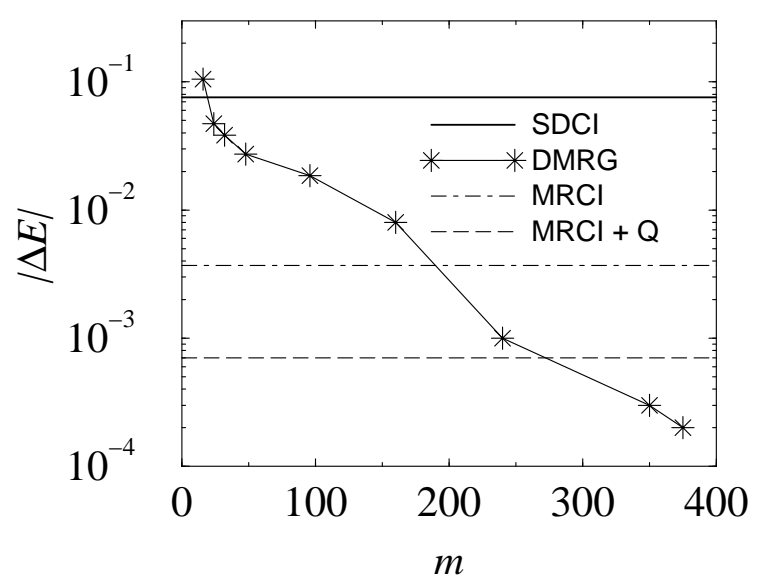

FIG. 3. Absolute value of the difference between the exact ground state energy within the given basis, and the approximate energy for various approaches, measured in Hartrees. The system is a water molecule with $\mathrm{OH}$ bonds stretched to twice their equilibrium length. The energy for MRCI+Q is below the exact result.

Hartree Fock gives a reasonably adequate description of a water molecule in its equilibrium geometry, but does not describe the system well when one of the $\mathrm{OH}$ bonds is stretched significantly. Many approximate approaches (such as SDCI) are strongly dependent on the adequacy of the HF starting point. In order to test the dependence of DMRG on the quality of HF, we have also studied the water molecule with both $\mathrm{OH}$ bond lengths doubled. This case was also studied by Bauschlicher and Taylor. In Fig. 3, we compare DMRG with SDCI, MRCI, 
and MRCI+Q. In this case, DMRG performs better than SDCI starting much earlier, $m \approx 20$. Both the MRCI and the DMRG results are largely unaffected by the poor HF starting point. In this case, the most accurate DMRG result is off by 0.00019 Hartrees, while the MRCI+Q result is off by 0.00071 Hartrees. Interestingly, both results are more accurate than for the equilibrium geometry. One can see that the convergence of DMRG appears to be roughly exponential in $m$, which is also usually the case in finite lattice model systems.

The numerical effort expended for these DMRG calculations was probably more than for the other approximate methods considered above, but less than for the full CI calculations. The point of our calculations was not to present a fully developed technique, but to demonstrate the potential of a new type of approach. DMRG is a versatile technique, and we believe substantial improvements can be made over the calculations described here. We will now discuss two general directions we believe could be successful in improving the method.

An area for potential progress is in the choice of a single particle basis. The Hartee Fock wavefunctions used here are reasonable choices for a small molecule: this basis gives a reasonable answer even if only one state per block is kept, $m=1$. Within the HF basis, the occupancy of orbitals tends to be either almost 0 or almost 2 , which helps the convergence of DMRG as a function of $m$. One could also try natural orbitals, which have similar properties. However, both HF and natural orbitals have a significant flaw: they are delocalized on a large system. Experience on lattice models suggests that DMRG tends to be much more accurate with localized bases. An ideal basis would be one that has occupancies close to 0 or 2, but would also be as local as possible. The DMRG would thus seem to fit naturally into the local correlation approaches currently being developed [9].

Another area for potential progress is in the grouping of similar orbitals into clusters. In the standard version of DMRG, blocks are formed for either the left or right half of the system. However, it is also possible to form blocks built out of clusters of orbitals which are strongly coupled to each other. This sort of procedure was found to be quite effective in an electron-phonon model, where a local density matrix was used to reduce the size of the local phonon space for each oscillator from up to 128 states to only 2 or 3 8. Here, one could form a cluster out of one "occupied" orbital plus a group "virtual" orbitals which are used to correlate the pair of electrons in the occupied orbital. One would want to organize the basis set into a set of such clusters, with each cluster having one occupied and a set of closely coupled virtual orbitals. A density matrix would be used to form an accurate many body basis for each of these clusters. Our preliminary calculations using this approach suggest that fewer than 50 states would be sufficient to describe such a cluster to millihartree accuracy, and even using just a few states would probably be a substantial improvement over Hartree Fock. (The Generalized Valence Bond approach [10] is closely related to this idea with $m=2$.) A standard DMRG calculation could then procede using these clusters as "sites", with the expectation that much higher accuracy for a given $m$ would be obtained than in the above orbital-by-orbital approach. Alternatively, one might group these clusters into superclusters, describing shells, atoms, or even molecules. In calculating the potential between two molecules, for example, it would be particularly natural to use a supercluster for each molecule. Note that efficient clustering would require the use of localized orbitals, so that progress in these two areas may be coupled.

We acknowledge support from the NSF under Grant No. DMR-9509945 (SRW), and the DOE/LDRD program at Los Alamos(RLM).

[1] S.R. White, Phys. Rev. Lett. 69, 2863 (1992), Phys. Rev. B 48, 10345 (1993).

[2] In DMRG, for a 1D system with short ranged interactions, the calculation time for a system of length $L$, keeping $m$ states per block, is proportional to $m^{3} L$. The error in the energy of the ground state is believed to vary as $\exp (-\alpha m)$ for systems with a gap to excited states. Since any finite system has gaps, we expect to get exponential convergence asymptotically for any finite system. However, the convergence using the infinite system DMRG method in a gapless system is generally not exponential, although it is usually still rather rapid.

[3] G. Fano, F. Ortolani, and L. Ziosi, preprint, condmat/9803071.

[4] Another difference is that this Hamiltonian is not at all one dimensional, which decreases the accuracy of DMRG. However, the correlations within an atom or small molecule are quite different from in a model system: for example, the Hartree Fock configuration is a much larger component of the ground state in a molecule. In fact, numerical renormalization group methods were originally developed to deal with a wide range of energy scales, as found in molecular species.

[5] Here, for simplicity, the two sites between the two blocks in the standard superblock configuration are assumed to be parts of their neighboring blocks.

[6] T. Xiang, Phys. Rev. B 53, 10445 (1996).

[7] C. W. Bauschlicher and P. R. Taylor, J. Chem. Phys. 85, 2779 (1986).

[8] C. Zhang, E. Jeckelmann, and S. R. White, Phys. Rev. Lett. 80, 2661 (1998).

[9] A. E. Azhary, G. Rauhut, P. Pulay and H. J. Werner, J. Chem. Phys 108, 5185 (1998), and references therein.

[10] F.W. Bobrowicz and W.A. Goddard III, in Methods of Electronic Structure Theory, H.F. Schaefer III, ed., Plenum, New York(1977). 